\author{
Rahul K. Ambekar* and Uttam D. Kolekar
}

\title{
FL-TOHIP (Fractional Lion Algorithm to Topology-Hiding Multipath Routing Protocol) in Mobile ad hoc Network
}

DOI 10.1515/jisys-2016-0178

Received August 5, 2016; previously published online April 4, 2017.

\begin{abstract}
Due to the dynamic nature of the mobile ad hoc network (MANET), all the nodes are mobile and generously connected to other nodes without the use of any fixed communications. Because of the random movement of nodes, the network topology is not predicted easily. Therefore, routing is one of the major challenging tasks in MANET. Significantly, the topology-hiding multipath routing protocol (TOHIP) is used to eliminate the topological exposure problem by transmitting the information through disjoint paths from the source to the destination. To select the optimal routing path, we have proposed the fractional lion algorithm to TOHIP (FL-TOHIP). Initially, the routing path is designed in MANET based on the existing TOHIP, which generates the number of disjoint routing paths. Then, the optimal path selection is done by the proposed fractional lion algorithm. To improve the solution searching, the fractional lion algorithm is adapted to the mathematical theory called fractional calculus. Finally, the performance of the proposed protocol is compared to the existing protocol using the delay, energy, and cost.
\end{abstract}

Keywords: Mobile ad-hoc network, topology-hiding multipath routing protocol, fractional lion algorithm, optimal routing path, disjoint routing path.

\section{Introduction}

In network topology, routing $[1,4,6,9,12-17,19-23,25,26]$ is defined as the transfer of information from a source to a destination. The objective of the routing process is used to discover routes and relocate as many data-packets as possible with the lowest routing operating cost [2]. During packet transmission, several problems may occur, such as delay, jitter, and loss of packets. To deal with these problems, some extra efforts need to be taken in highly dynamic mobile ad hoc networks (MANET) [7, 8]. Based on the structure of the network, the routing protocol of the MANET is classified into three parts: geographic location-assisted routing, horizontal routing, and hierarchical routing [18]. In MANET, the accumulation and removal of nodes are carried out due to the mobility of nodes. Hence, unexpected variations occurred in the network topology. Many different protocols have been proposed to resolve the routing problems in ad hoc networks, which are roughly classified as unicast protocol, multicast protocol, broadcast protocol, and anycast protocol. The unicast protocol is also called as one-to-one protocol, in which the data packets are transferred to an exact destination [24]. Then, the packet is transmitted to all members of the exacting group [3] in the multicast protocol. The reliability of data transmission and bandwidth is increased by the multipath routing [1]. However, the congestion problem is increased in the multipath routing and the cost of the router also gets increased in the multipath routing [13]. Due to the random nature of mobile nodes in MANET, channel bandwidth and battery power are limited. Due to these limitations, MANET creates a link, node susceptibility, and unsteadiness when data communication happens.

*Corresponding author: Rahul K. Ambekar, Pacific Academy of Higher Education and Research University, Udaipur, Rajasthan 313003, India, e-mail: rahulambekar0103@gmail.com

Uttam D. Kolekar: A.P. Shah Institute of Technology, Thane West, Thane, Maharashtra 400615, India 
During packet transmission, various problems occur in the network, such as delay, jitter, and packet loss, which brutally affects the performance of the network if the appropriate path for communication is not determined. The transmission time of the packets is getting increased due to the unavailability of network resources, which is called delay. Then, the difference in the end-to-end delay of packets traveling from the source to the destination is measured as jitter and the rate of loss of transmission packets is measured as packet loss. In [21], the ant colony algorithm was used to find the routing path for the mobile nodes. The ant colony explores the search space without initial directions, which leads to the risk of having local optima; also, the objective of finding the multipath does not consider the multiple constraints, such as distance, energy, and delay within the algorithm [21]. In [15], the hybrid routing algorithm was designed by combining the ant colony and particle swarm optimization (PSO) algorithms for routing in MANETs. Here, serial hybridization was proposed to find the feasible path for the data transmission without mainly considering the end-to-end delay, power consumption, and communication cost when routing a packet from a source to a destination. In [25, 26], the topology-hiding multipath routing protocol (TOHIP) was proposed to handle the topology exposure problem in MANET. These works make use of the route request phase additionally to hide the network topology while sending the data packets. However, the dynamic grouping based on the clustering process may improve the security concern due to the dynamic change of routing information.

The primary intention of this research is to design and develop a TOHIP for data transmission in MANET. The routing protocol is designed based on the recent optimization algorithm called lion algorithm [20]. Then, the proposed multipath routing protocol is designed based on the new optimization algorithm called fractional lion (FL), which is newly proposed by combining the fractional theory and lion algorithm. Also, to alleviate the identified problems, new objective constraints are mathematically modeled based on different parameters, such as delay, energy, and distance, which are then used by the fractional lion algorithm to find the optimal multipath for reliable data transmission in MANETs. The proposed routing protocol is ensuring the shortest distance, minimum delay, low power consumption, and low cost when compared to the existing TOHIP. Also, the proposed algorithm does not use the routing information within the message to handle the topology exposure problem. The performance of the proposed protocol is analyzed using throughput and energy.

The main contributions of the paper are discussed as follows:

1. The first contribution of the paper is an optimal path selection from the number of generated disjoint routing paths using the proposed FL-TOHIP. In this paper, we have designed the new objective function to evaluate the optimal path selection process. Here, the selection of optimal routing path considered is based on the four different kinds of objectives: throughput, delay, energy consumption, and packet drop. Finally, the proposed routing protocol is ensuring the shortest distance, minimum delay, low power consumption, and low cost when compared to the existing TOHIP.

2. The second contribution is an optimization process using the mathematical model of fractional calculation with lion algorithm. Fractional calculus is used to improve the solution search in the predefined search space based on the user requirement. To improve the solution search in the optimal routing path, we have used the lion algorithm with fractional calculus.

The paper is organized as follows: Section 2 presents the literature survey of various routing protocols with their merits and demerits. Then, Section 3 presents the problem statement based on the routing protocol. Section 4 describes the detailed explanation of the proposed FL-TOHIP in MANET. Section 5 presents the experimental results and Section 6 concludes the paper.

\section{Related Work}

This section discusses various topological routing protocols in MANET for transmitting the data from the source to the destination $[4,6,9,15,17,19-22,25,26]$. Zhang et al. [26] demonstrated the robust routing 
protocol called TOHIP (THMR), which has been limited in performance based on the delay. Additionally, Sharma et al. [19] have presented an ad hoc on-demand distance vector routing (AODV) protocol to transmit the packets from the source to the destination. Here, the developed AODV protocol is much more stable and better in terms of power conservation and network lifetime and is more energy efficient. However, the data packets may be delivered to too many nodes that do not need to receive them and potentially lower the reliability of data delivery. Singh et al. [21] have designed orientation based on the ant colony algorithm, in which the number of data packets transmitted is increased with low jitter and high throughput as well as maintaining less path length. However, the orientation method provides lesser efficiency in terms of security and routing. To improve the packet delivery ratio and path success ratio, Budyal et al. [6] have developed adaptive neuro-fuzzy inference system. Due to a reduction in end-to-end delay and overhead, it achieves higher packet delivery ratio. This method is not applicable for multiple anycast routes for different QoS requirements.

Taheri et al. [22] have proposed an anonymous group-based routing protocol to improve the privacy aspects of the multicast protocol. However, this system does not consider the cases in which more than one leader emerges in the network and a submesh group communication is formed. Then, the efficient algorithm is developed by Singh et al. [20], named as ant colony algorithm, which is highly suitable for finding the adaptive routing for such type of volatile network. However, the next hop count is increased when all the values are presented in the column. To provide a better multicast routing scheme, Biradar et al. [4] have developed the neighbor node selection method. Based on a threshold value, it selects the reliable neighbor node for transmission. The developed multipath routing requires information exchange between the nodes, which introduces the receiver complexity.

The existing methods addressed the following challenges such as delay in the transmission, problems with the packet delivery, reliability, security, and receiver complexity. These challenges have been considered in the proposed method called fractional lion optimization, which provides the secure TOHIP in MANET. The important aspect of the proposed method is that it can ensure the high-level security to the information without much packet loss.

\section{Problem Statement}

Figure 1 shows the topological structure of MANET. Here, the multiple nodes present in the MANET are denoted as black color in round shape. Then, the edges between the two nodes are represented by dotted lines. The nodes present in the MANET are mobile in nature, which have been characterized by the multihop

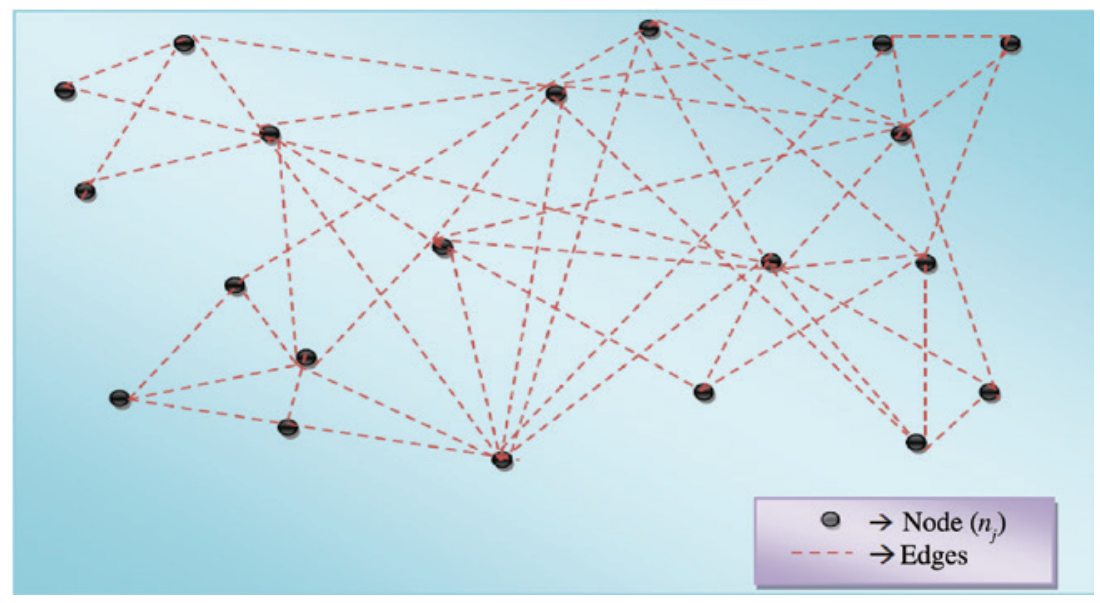

Figure 1: MANET Topological Structure. 
network topology. Due to their mobile nature, all the nodes are connected freely to other nodes in its range to transmit the information. The structure of entire MANET is represented as follows:

$$
M=\left\{n_{j}, 1 \leq j \leq N\right\}
$$

where $M$ is defined as the topological structure of MANET, $N$ denotes the total number of nodes present in the MANET and $n_{j}$ is the $j$ th number of node present inside the MANET. The numbers of nodes present inside the MANET are located in the form of coordinates. For example, the $j$ th number of nodes present inside the MANET is located in $\left(x_{i}, y_{j}\right)$ coordinates in the space.

\section{Problem 1: topology exposure problem}

Nowadays, one of the most serious problems presented in the MANET is named as topology exposure, which mainly affects the multipath routing protocols rather than the other routing protocols. To find the sufficient routes, the multipath routing protocol carries a lot of routing information in route messages. Therefore, the routing path information is known by the source node before transmitting the information. By capturing the routing information, the malicious nodes are generated to deduce the whole network topology. To explain this topological exposure problem, two nodes have been selected from the MANET topological structure, which have been denoted as $n_{i}$ and $n_{j}$. Then, the information is transferred from node $n_{i}$ to node $n_{j}$ through a more number of intermediate nodes. While transferring the information from $n_{i}$ to $n_{j}$ with the routing message, node $n_{i}$ may find the nodes that are connected to node $n_{j}$. The routing path between the two nodes $n_{i}$ and $n_{j}$ can be represented as follows:

$$
r\left(n_{i}, n_{j}\right)=\left\{n_{i}, \cdots \cdots, n_{j}\right\} ; 1 \leq j \leq N ; 1 \leq i \leq N
$$

Here, node $n_{i}$ is not as same as node $n_{j}$, which is denoted as $i \neq j$. Here, the number of routing paths is available between the source and destination node. To deal with this topological exposure problem, the TOHIP does not contain any routing information while transferring the information. Here, the distance is calculated between the two nodes $n_{i}$ and $n_{j}$. Within two hops at most, the network topology can be deduced by any nodes in MANET. When the distance between these two nodes is greater than $2\left(\operatorname{Dist}\left(n_{i}, n_{j}\right)>2\right)$, node $n_{i}$ does not know about the nodes that are connected to node $n_{j}$. Here, both nodes $n_{i}$ and $n_{j}$ belong to the total number of nodes present inside in the MANET $\left(n_{i}, n_{j} \in N\right)$.

\section{Problem 2: selection of optimal routes}

In [26], the TOHIP is used to find the routing path based on the source information. At the end of this routing process, the numbers of disjoint paths are generated that can be represented as follows:

$$
R\left(n_{i}, n_{j}\right)=\left\{r_{1}\left(n_{i}, n_{j}\right), r_{2}\left(n_{i}, n_{j}\right), r_{3}\left(n_{i}, n_{j}\right), \cdots \cdots, r_{k}\left(n_{i}, n_{j}\right)\right\}
$$

where $k$ is the total number of generated disjoint paths and $r_{x}\left(n_{i}, n_{j}\right)$ is the $x$ th number of routing path available between the two nodes $n_{i}$ and $n_{j}$. From these generated numbers of disjoint paths, the user needs to select the optimal path based on the less delay, conserved energy, and less routing distance. To select the optimal routing path, we have proposed a fractional lion algorithm in this paper.

\section{Protocol Design: FL-TOHIP in MANET}

In this section, Figure 2 shows the proposed routing protocol design based on the fractional lion optimization algorithm. The steps involved in the proposed routing protocol design are discussed as follows: Initially, the original MANET topology such as in Figure 1 is applied through the TOHIP to find the routing path between the source and destination node. Here, the TOHIP is used to generate the number of disjoint paths from the source node to the destination node and removing the redundant paths before transmitting the information. 


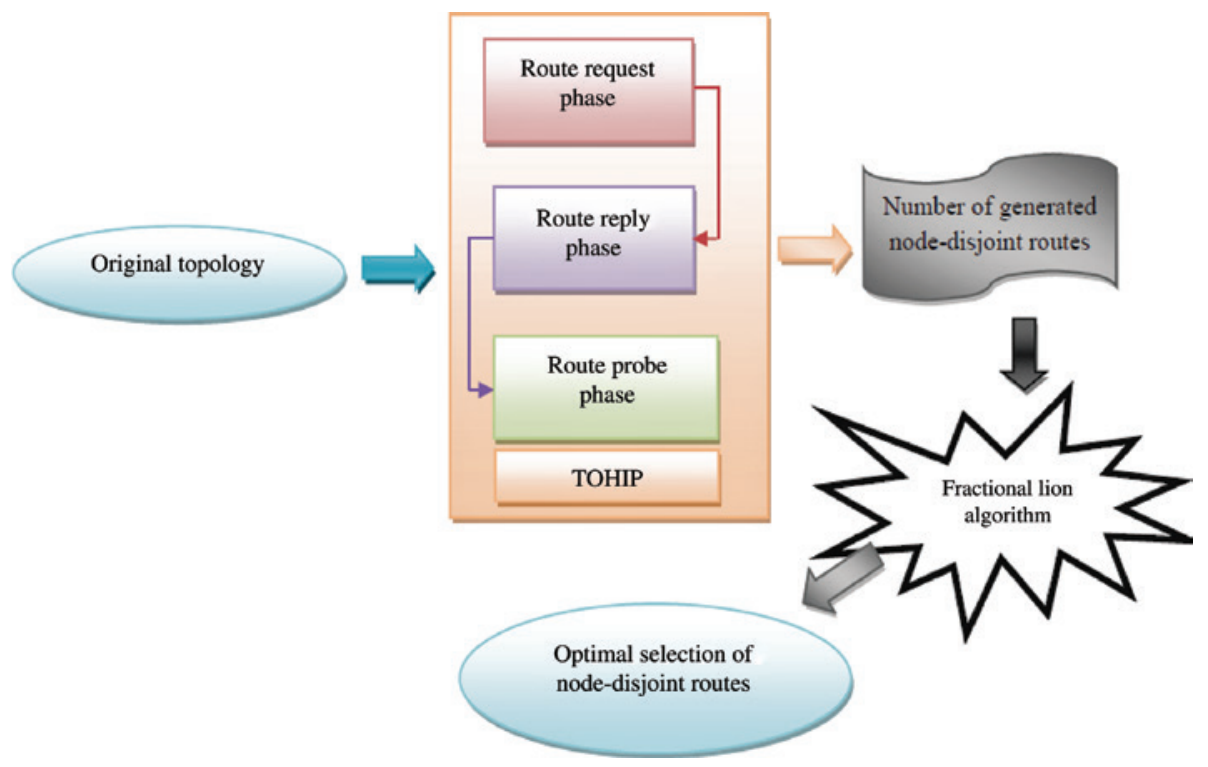

Figure 2: Block Diagram Representation of Proposed Routing Protocol.

Then, the optimal path selection is done with the results of the TOHIP using the proposed fractional lion algorithm.

\subsection{TOHIP Algorithm for Multipath Route Discovery}

This section presents the detailed discussion of TOHIP [26]. To find the routing path from the source to the destination, the TOHIP is used. Initially, two nodes are selected from the MANET as the source and destination, which have been denoted as $n_{i}$ and $n_{i}$. Here, the TOHIP algorithm is used to transfer the information from node $n_{i}$ to node $n_{j}$ using effective routing paths without considering the redundant paths. The main objective of designing TOHIP is described as follows: (i) The malevolent nodes cannot deduce the network topology due to the hidden format of link connection information. (ii) The reliable packet delivery and load balancing is achieved and (iii) used to detect unreliable nodes before transmitting the information. Then, the process of the TOHIP is performed by three phases named as route request phase, route reply phase, and route probe phase. Then, the process of each phase can be explained as follows.

\subsubsection{Route Request Phase}

In the route request phase, the information is transmitted with the route request message (RREQ) from a source to a destination. To maintain the network connectivity, the receiving node sends an acknowledgment to the sender node after receiving the request message. For example, one network topology contains 14 nodes, which have been denoted as $n_{i}, n_{l}, n_{m}, n_{n}, n_{f}, n_{h}, n_{b}, n_{e}, n_{d}, n_{g}, n_{y}, n_{c}, n_{a}$, and $n_{j}$. From these 14 nodes, we have taken $n_{i}$ as the source node and $n_{j}$ as the destination node. Initially, the information is transferred from source node $n_{i}$ to destination node $n_{j}$ using the RREQ $\left\langle n_{i}, n_{j}\right.$, Seq, Hct $\left.=0\right\rangle$ through intermediate nodes such as $n_{f}, n_{h}$, $n_{b}, n_{e}, n_{d}, n_{g}, n_{y}, n_{a}$, and $n_{c}$. Here, $S_{e q}$ denotes the sequential number, which is defined by source node $n_{i}$. For example, we have taken $n_{a}$ as an intermediate node, which has five numbers of neighboring nodes named as $n_{b}, n_{c}, n_{e}, n_{f}$, and $n_{g}$. At first, the RREQ is received by node $n_{a}$ from node $n_{g}$. Then, node $n_{a}$ inserts that RREQ message inside the sequence number table as $\left\langle n_{i}\right.$, Seq $\rangle$ and also stores the RREQ message inside the routing table as $\left\langle n_{i}, n_{j}\right.$, Hct +1$\rangle$. During this route request phase, the sequence number table is generated by each node, which is used to prevent the rebroadcasting of that particular node from unnecessary route messages. 


\subsubsection{Route Reply Phase}

Once the route request phase is completed, destination $n_{j}$ receives the RREQ copy and broadcasts it using the route reply phase. During the process of route reply phase, the routing message contains various parameters such as source ID, destination ID, number of hop count to the destination node, and next nodes consideration and excluded node set generation. Here, the route reply message is initiated as $\left\langle n_{i}, n_{i}, \mathrm{Hct}=0, \operatorname{EXN}\left\{n_{b}, n_{c}\right\}, \mathrm{NXN}=\right.$ null $\rangle$, where EXN and NXN are defined as the excluded nodes and the neighboring nodes, respectively. The next node is generated based on the least number of hops present in the routing path. Due to the consideration of hop count, we can avoid the wormhole attackers on the particular route. For example, node $n_{a}$ receives the RREQ message as $\left\langle n_{i}, n_{j}, \operatorname{Hct}=1, \operatorname{EXN}\left\{n_{b}, n_{c}, n_{a}\right\}, \mathrm{NXN}=n_{a}\right\rangle$ from node $n_{b}$. At the end of this route reply phase, node $n_{a}$ removes the excluded routes as $n_{b}, n_{c}$, and $n_{a}$ and the shortest path node is selected from the neighboring nodes. Finally, the numbers of disjoint routing paths are created from source node $n_{i}$ to destination node $n_{j}$, which can be described as follows:

$$
R\left(n_{i}, n_{j}\right)=\left\{r_{1}\left(n_{i}, n_{j}\right), r_{2}\left(n_{i}, n_{j}\right), r_{3}\left(n_{i}, n_{j}\right), r_{4}\left(n_{i}, n_{j}\right)\right\}
$$

where $k$ is the total number of disjoint routing path and $u$ denotes the number of routing paths available between the source and destination node. Here, the intermediate nodes present in the routing path $r_{1}\left(n_{i}, n_{j}\right)$ are denoted as $n_{i} \rightarrow n_{y} \rightarrow n_{f} \rightarrow n_{c} \rightarrow n_{j}$. In the same way, the intermediate nodes present in the routing path $r_{2}\left(n_{i}, n_{j}\right)$ are represented as $n_{i} \rightarrow n_{g} \rightarrow n_{a} \rightarrow n_{b} \rightarrow n_{j}$. In addition, the intermediate nodes present in the routing path $r_{1}\left(n_{i}, n_{j}\right)$ are described as $n_{i} \rightarrow n_{h} \rightarrow n_{e} \rightarrow n_{d} \rightarrow n_{j}$ and the disjoint routing path of $r_{4}\left(n_{i}, n_{j}\right)$ is created as $n_{i} \rightarrow n_{l} \rightarrow n_{m} \rightarrow n_{n} \rightarrow n_{j}$.

\subsubsection{Route Probe Phase}

Once the disjoint paths are generated, the information is transmitted through the generated paths from the source to the destination. Before transmitting the information, the route probe message is initiated by source node $n_{i}$ and transferred to the destination node that has been established in route reply messages. The main use of route probe usage is discussed as follows: (i) The unreliable routes are detected and removed by the route probe phase. (ii) The route probe phase finds the secure and shortest routing path. If the disjoint routing path $r_{4}\left(n_{i}, n_{j}\right)$ is detected as an unreliable routing path, the particular detected path is removed. Then, the source transmits the information through the remaining paths of $r_{1}\left(n_{i}, n_{j}\right), r_{2}\left(n_{i}, n_{j}\right)$, and $r_{3}\left(n_{i}, n_{j}\right)$. Finally, the information is sent through the three routing paths that can be represented as follows:

$$
\begin{gathered}
R_{d}\left(n_{i}, n_{j}\right)=r_{1}\left(n_{i}, n_{j}\right), r_{2}\left(n_{i}, n_{j}\right), \cdots, r_{d}\left(n_{i}, n_{j}\right) \\
r_{1}\left(n_{i}, n_{j}\right) \Rightarrow n_{i} \rightarrow n_{y} \rightarrow n_{f} \rightarrow n_{c} \rightarrow n_{j} \\
r_{2}\left(n_{i}, n_{j}\right) \Rightarrow n_{i} \rightarrow n_{g} \rightarrow n_{a} \rightarrow n_{b} \rightarrow n_{j} \\
r_{3}\left(n_{i}, n_{j}\right) \Rightarrow n_{i} \rightarrow n_{h} \rightarrow n_{e} \rightarrow n_{d} \rightarrow n_{j}
\end{gathered}
$$

where $R_{d}\left(n_{i}, n_{j}\right)$ is defined as the collection of generated disjoint routing paths and $d$ denotes the total number of the disjoint routing path.

\subsection{Selection of the Optimal Number of Disjoint Routes using Fractional Lion Algorithm}

From the results of the TOHIP algorithm, we can obtain the multiple $(k)$ numbers of disjoint routing paths from the source to the destination. The selection of optimal disjoint path from these generated paths is one 
of the major challenging tasks in MANET. To deal with this challenge, we have proposed a new topological model called FL-TOHIP, in which the fractional lion algorithm is used for fitness function using the parameters of energy, delay, and distance.

\subsubsection{Solution Encoding}

In the solution encoding process given in Figure 3, we have developed efficient encoding solutions that have been used to reduce the searching space significantly. To achieve this, the solution vector is generated based on the user requirement. For example, $k$ numbers of disjoint routing paths $R_{k}\left(n_{i}, n_{j}\right)$ are selected from the MANET using the existing TOHIP. The selection of optimal routing path $R_{q}\left(n_{i}, n_{j}\right)$ from the generated disjoint routing paths $R_{k}\left(n_{i}, n_{j}\right)$ is based on the user requirement, which is one of the major challenging tasks considered in this paper. Here, the value of $q$ is always lesser than the value of $k$. Initially, the number of optimal paths is not known by the user. To select the optimal path, we have proposed the fitness measurement.

\subsubsection{Fitness Function}

The fitness function is defined as an objective function that is used to summarize the performance of the given design solution according to the target. The main intention of this research is to find out the optimal routing path for reliable data transmission in MANETs. To generate the targeted output, we have developed the fitness function formula in this paper. Here, the fitness function is measured based on the reduced form of delay, energy, and distance between the nodes. The proposed fitness function is described as follows:

$$
\begin{gathered}
\text { Fitness }(F)=\sum_{i=1}^{k} \sum_{j=1}^{N_{i}} f_{i j} \\
f_{i j}=\left(\frac{f_{i j}^{D}+f_{i j}^{E}+f_{i j}^{d}}{3}\right)
\end{gathered}
$$

where the total number of selected optimal disjoint routing path vector is denoted as $k$, and $i$ denotes the number of selected optimal disjoint routing path vector. Then, the number of nodes present inside the selected optimal routing path is denoted as $N_{i}$. At first, the distance between the nodes is calculated in the routing path using the formula of $f_{i j}^{D}$. From this calculation, we can find the optimal routing path based on the shortest distance path calculation:
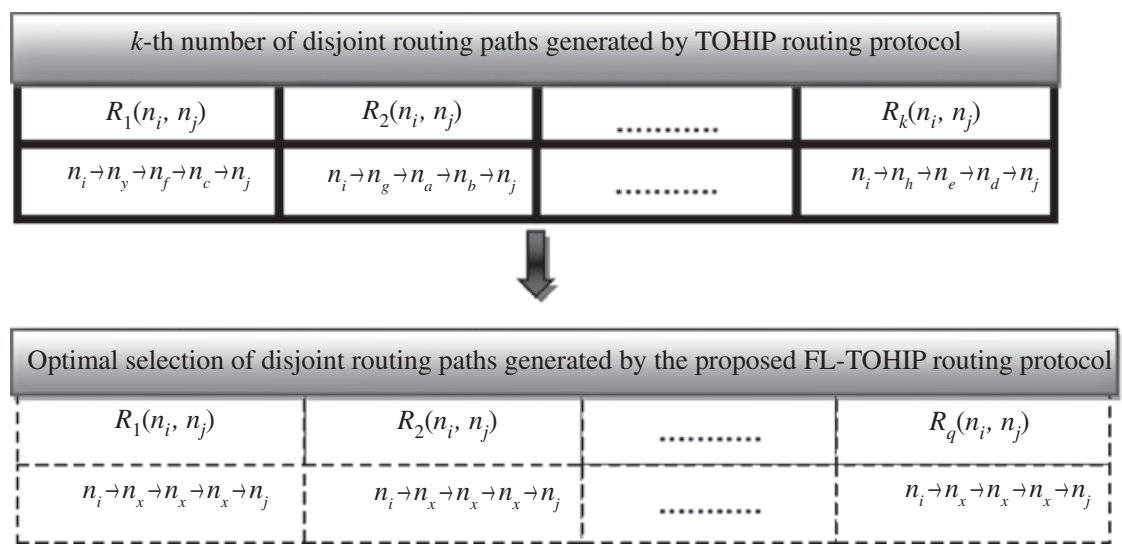

Figure 3: Solution Encoding. 


$$
f_{i j}^{D}=\frac{1}{\left(N_{i}-1\right) \mathrm{MD}} \sum_{k=1}^{N_{i}-1} \operatorname{Dist}(k, k+1)
$$

where the distance calculation of the routing path is measured as $f_{i j}^{D}$ and $\operatorname{Dist}(k, k+1)$ is defined as the distance between the two nodes, which is present inside the particular disjoint routing path. MD denotes the maximum distance calculation from the source node to the destination node. Second, the energy consumption between the nodes is considered as the fitness measurement, which can be described as follows:

$$
f_{i j}^{E}=\left(1-E\left(N_{j}\right)\right)
$$

The total energy consumption due to the selected disjoint routing path can be represented as $f_{i j}^{E}$. Basically, the highest energy consumption between the nodes leads to reduce the lifetime of the MANET. To deal with this drawback, we have selected the optimal routing path based on the lowest energy consumption. Finally, the delays of the routing nodes are considered as the fitness evaluation to reduce the transmission time of the routing protocol. When the distance between the nodes increases, the transmission speed also is reduced. Due to the reduction of transmission speed, the delay also gets reduced. To reduce the transmission delay, the fitness measurement is developed, which can be expressed as follows:

$$
f_{i j}^{d}=\frac{f_{i j}^{D} \times N_{j}}{n}
$$

where $f_{i j}^{D}$ is defined as the calculation of delay between the nodes, $n$ is the total number of nodes present in the MANET, and $N_{j}$ is the $j$ th number of nodes present in the disjoint routing path.

\subsubsection{FL-TOHIP: Proposed Optimization Algorithm}

To perform the routing of packets with high energy efficiency, less delay, and high throughput, the optimization-based routing protocol is used. To perform the optimization, we have used lion algorithm in this paper. The lion algorithm [16] is one of the recently introduced optimization algorithms that are used to accomplish the system characteristics accurately based on the lion's unique characteristics. When the selected solution is equal to the random solution, the problem occurs in the lion algorithm. To overcome this problem, we have proposed fractional calculus [10,11] with the lion algorithm. The extension of conventional mathematics is defined as fractional calculus, which consists of irreversibility and inherent memory property to renew the values dynamically. The steps involved in the searching process of the proposed fractional lion algorithm can be represented as follows:

1. Initially, three vectors are randomly generated by the user named as $X_{m}, X_{f}$, and $X_{n}$. The size of the randomly generated vectors is based on the size of the optimal routing path.

2. Then, fitness measure $X_{r}$ is calculated using the newly developed fitness formula $F$.

3. The fertility evaluation process is carried out to avoid the converging in the local optima. Here, fertility evaluation is based on the following parameters: sterility rate $\left(s^{r}\right)$, female update count $\left(u^{c}\right)$, female generation count $\left(G^{c}\right)$, and laggardness rate $\left(l^{r}\right)$. From the results of fitness value, two solution vectors are generated such as male $N_{1}$ and female $N_{2}$ vectors. Here, the reference value is less than the value of the solution vector $f\left(X_{r}\right)<f\left(X_{m}\right)$. We can consider the final solution vector as $X_{m}$. Then, this process is continued based on the laggardness rate value. Similarly, female vector $X_{f}$ is also calculated based on the sterility rate.

4. Then, the mating process is carried out by mutation and crossover between the two generated solution vectors as $X_{m}^{c}$ and $X_{f}^{c}$. From the results of the mating process, the cubs of both solution vectors are generated. 
5. After completing the mating process, the terrestrial defense and terrestrial takeover process are carried out. During the terrestrial defense, the existing solution vector is compared to randomly selected vector (nomadic vector). For example, the function of $X_{m}$ is greater than the function of $X_{n}$ and the subvectors of both $X_{m}^{c}$ and $X_{f}^{c}$ are eliminated. Then, nomadic vector $X_{n}$ is replaced by male vector $X_{m}$. Then, the existing vector is replaced by a new one, when the new vector (nomadic vector) is better than the existing vector (male vector).

6. When the results of both the new and existing vectors are equal, the selection of the best vector from the new and existing vectors is one of the major challenging tasks in this algorithm. To overcome this challenge, we have proposed fractional calculus with the lion algorithm. When the functions of both $f\left(X_{m}\right)$ and $f\left(X_{n}\right)$ vectors are same, the solution of both vectors generates the same value, which can be represented as follows:

$$
X_{m}^{t+1}=X_{m}^{t}
$$

where $X_{m}^{t+1}$ is defined as the new vector generated from the lion algorithm and the existing solution in the lion algorithm can be represented as $X_{m}^{t}$. To modify the order of solution equation, the results of $X_{m}^{t+1}$ is rearranged in the following manner:

$$
X_{m}^{t+1}-X_{m}^{t}=0
$$

Here, $X_{m}^{t+1}$ is considered as the discrete version of the derivative of $\alpha_{F}=1$, which leads to the following equation:

$$
D^{\alpha_{F}}\left[X_{m}^{t+1}\right]=0
$$

To select the optimal path, the value of $\alpha_{F}$ can be changed from 0 to 1 . Then, the flat variation and longer effect of memory can be maintained by fractional calculus observation. Then, the above equations can be written by considering the second-order derivative functions can be represented as follows:

$$
\begin{gathered}
X_{m}^{t+1}-\alpha_{F} X_{m}^{t}-\frac{1}{2} \alpha_{F} X_{m}^{t}=0 \\
X_{m}^{t+1}=\alpha_{F} X_{m}^{t}-\frac{1}{2} \alpha_{F} X_{m}^{t}
\end{gathered}
$$

where $X_{m}^{t+1}$ is defined as the final solution vector generated from the fractional calculus method.

7. Finally, the solution vector generated by fractional calculus $X_{m}^{t+1}$ is selected as $X_{m}^{\text {best }}$ by eliminating all other weak solutions.

\subsection{Packet Transmission using the Optimal Route}

The existing routing protocol [26] generates three disjoint routing paths to transmit the data packets. Based on this existing concept, several numbers of disjoint paths are developed for the large topological structure of MANET. Then, the selection of optimal path from this generated disjoint path is taken as the major challenge in this paper. To avoid this problem, we have proposed the fractional lion routing protocol, in which the packet transmission is done using the optimal routing path. The fractional lion algorithm chooses the optimal path from the generated disjoint routing path. In this paper, the optimal path selection is based on the less delay, conserved energy, and less distance routing path. To achieve this optimal path selection, the fitness measurement is proposed in this paper. Finally, the optimal path is selected between the sources to the destination to transmit the information. Then, the representation of optimal path generation can be expressed as follows: 


$$
R_{Q}\left(n_{i}, n_{j}\right)=r_{1}\left(n_{i}, n_{j}\right), r_{2}\left(n_{i}, n_{j}\right), \cdots, r_{q}\left(n_{i}, n_{j}\right)
$$

where $Q$ represents the total number of optimal path selected given by the user.

\section{Results and Discussion}

This section shows the simulation of MANET environment and the experimental results of the proposed FLTOHIP method for optimal path selection. Then, the proposed FL-TOHIP is compared to the TOHIP using the parametric analysis, which has been performed based on the delay, energy, packet loss, and throughput.

\subsection{Simulation Setup}

In this paper, we have used MATLAB software to perform the simulation of MANET using various numbers of nodes. The reason for selecting the MATLAB to perform the simulation is that MATLAB can provide the expected simulation and it would be easy to implement the algorithms compared to other tools, namely, ns-1, ns-2, and ns-3. The ns-2 simulator is not based on real-time scenario and the effort for simulating in the OMNeT++ is large when compared to the other simulators. Moreover, OPNET has the capacity only to store a single event list at a time and the event is cleared after its execution is completed. On the contrary, MATLAB is a powerful tool that saves time and reduces the computational complexity. MATLAB enables the immediate testing of the algorithms without any recompilation and it provides immediate access to a large number of functions. MATLAB possesses the capacity to store a large number of databases unlike the other simulators, which suffer from workload. In the simulation, the MANET nodes are fixed in the area of $100 \times 100$ and $50 \times 50 \mathrm{~m}$. Then, the performance evaluation is done based on the evaluation metrics. Thus, the simulation results of proposed method and its values are tabulated in Table 1.

\subsection{Performance Evaluation}

The performance is evaluated based on four different metrics. The delay is the ratio of the time taken to reach the destination from the source. Throughput is defined as the rate of successful data transmission with respective particular time. Packet loss is used to measure the loss of packets during the transmission.

\subsubsection{Performance Evaluation Based on Throughput}

The throughput analysis of the proposed FL-TOHIP is shown in Figure 4. Figure 4A shows throughput results of MANET with a $50 \times 50 \mathrm{~m}$ area. Here, the rate of successful packet delivery is calculated between the two

Table 1: Simulation Parameters and Its Values.

\begin{tabular}{ll}
\hline Parameters & Values \\
\hline Simulation area & $100 \times 100 \mathrm{~m}$ \\
Number of mobile nodes & 50,100 \\
Simulation time & 10,20 \\
Packet generation rate & 1 packet $/ \mathrm{s}$ \\
Node movement speed & $12 \mathrm{~m} / \mathrm{s}$ \\
Number of attackers & 2 \\
Mobility model & Random \\
Antenna model & Omnidirectional \\
Energy model & Given in [5] \\
\hline
\end{tabular}




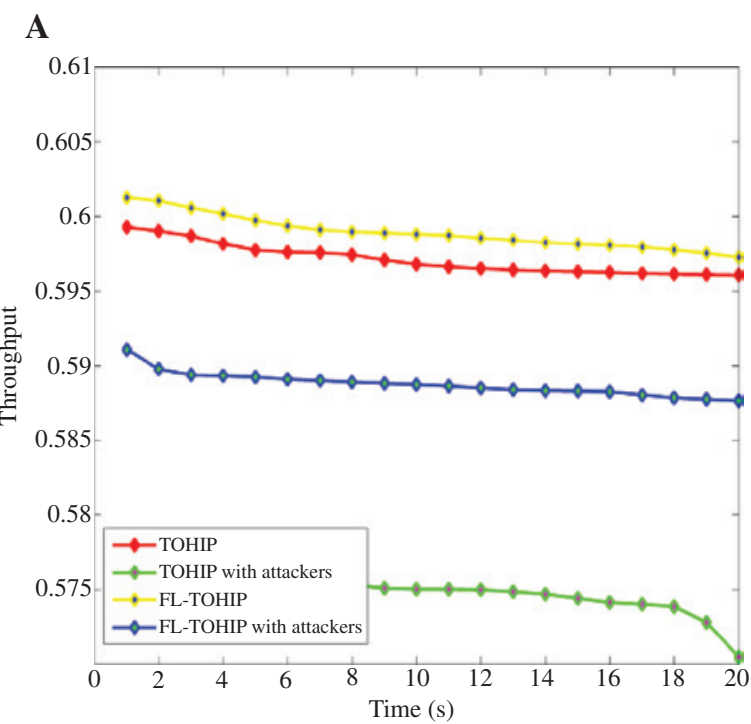

\section{B}

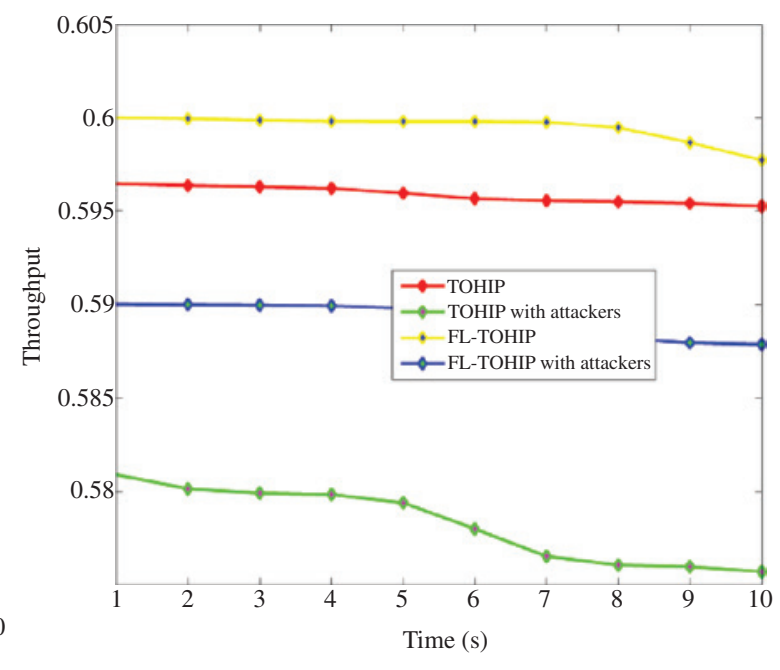

Figure 4: Throughput Analysis: (A) $t=20$ and Nodes $=50$ and (B) $t=10$ and Nodes $=100$.

routing protocols named as TOHIP and FL-TOHIP with and without the attacker nodes. Initially, the throughput value is calculated without any attackers present in the network. When the transmission time $(t)$ of data is fixed as $2 \mathrm{~s}$, the rate of successful packet delivery through the TOHIP routing path is obtained as 0.59 . At the same time, the throughput value of the proposed FL-TOHIP is achieved as 0.60. Further increasing the transmission time from 2 to $20 \mathrm{~s}$, the throughput of both TOHIP and FL-TOHIP is obtained as 0.596 and 0.597, respectively. Basically, the attackers present in the routing path are used to reduce the throughput value. Then, the throughput performance of the both FL-TOHIP and TOHIP is analyzed with the attackers. When the transmission time is fixed as $18 \mathrm{~s}$, the throughput of the TOHIP and the proposed FL-TOHIP is measured as 0.57 and 0.58 , respectively. From the above results, we can say the proposed FL-TOHIP transmits the number of data packets with high throughput.

Figure 4B shows the comparison result of both TOHIP and FL-TOHIP based on the throughput using the $100 \times 100$ m MANET area. At first, the evaluation of the packet transmission is done without using any attacker nodes. Here, when the time of data transmission is fixed as $4 \mathrm{~s}$, the throughput of the proposed FLTOHIP is achieved as 0.599. Meanwhile, the throughput of the TOHIP is obtained as 0.596. The attacker nodes reduce the rate of the successful transmission of the packets. Therefore, the throughput calculation needs to be analyzed using the attacker nodes. Initially, the transmission time is fixed as 2 , and the throughput measurement of the TOHIP is achieved as 0.58. Temporarily, the throughput value of the proposed FL-TOHIP is obtained as 0.59 , which is higher than the TOHIP. From this figure, we can conclude that the highest throughput is achieved by the proposed FL-TOHIP compared to the existing TOHIP.

\subsubsection{Performance Evaluation Based on Delay}

Figure $5 \mathrm{~A}$ shows the delay calculation of the proposed routing protocol compared to the existing TOHIP. When the time of data transmission is fixed as $2 \mathrm{~s}$, the TOHIP takes more time to transmit the packet when compared to the proposed FL-TOHIP. The delay of both TOHIP and FL-TOHIP is obtained as 0.241 and 0.240, respectively. Here, the delay of TOHIP delay is greater than the proposed routing protocol. Then, the performance of the proposed method is further analyzed with the attacker node. When the transmission time is fixed as $20 \mathrm{~s}$, the data transmission of the TOHIP is done with the delay of 0.263.

Figure 5B shows the delay calculation of the routing protocol by considering the $100 \times 100 \mathrm{~m}$ MANET area. When the data transmission time is fixed as $3 \mathrm{~s}$, the maximum delay is obtained by the TOHIP as 0.2377 . 

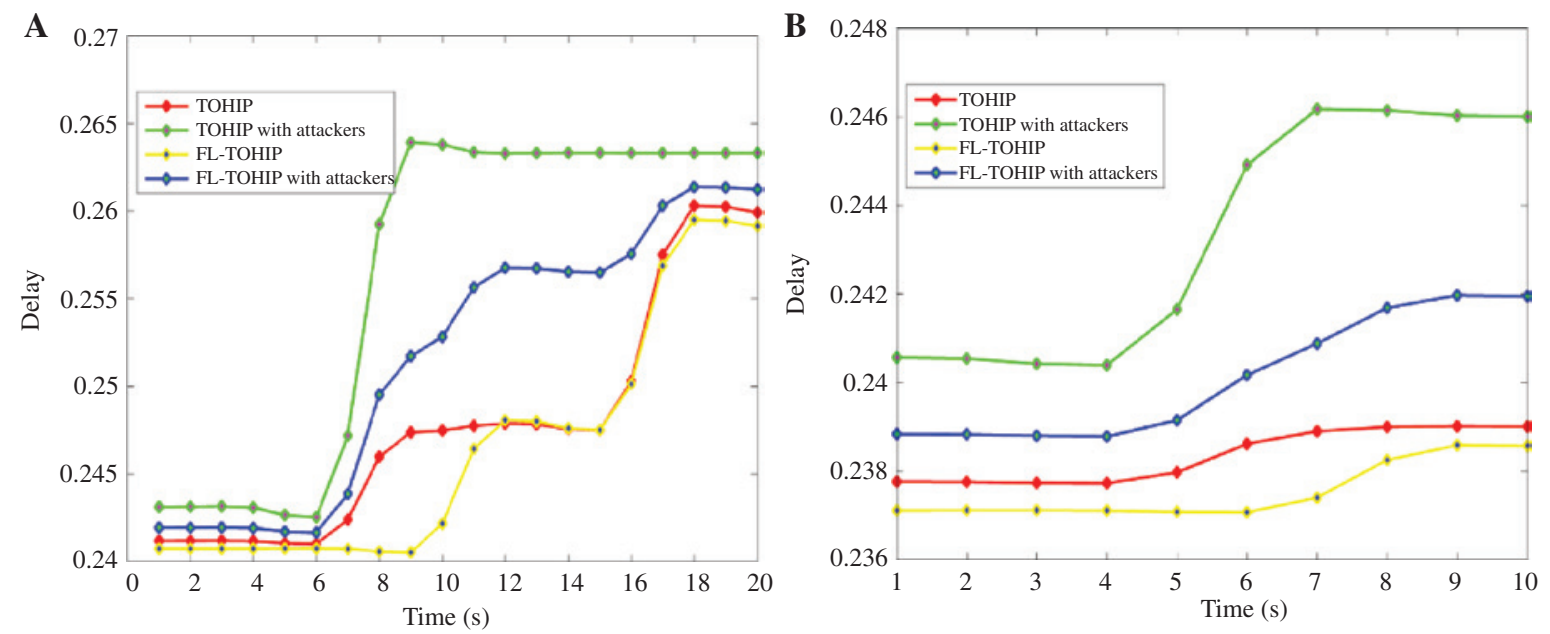

Figure 5: Analysis of Delay: (A) $t=20$ and Nodes $=50$ and (B) $t=10$ and Nodes $=100$.

At the same time, the proposed FL-TOHIP obtains the minimum delay as 2.3711. Then, the time of data transmission is increased from 3 to $6 \mathrm{~s}$. The delay value of the existing TOHIP is increased as 0.238. In the same way, the delay of the proposed FL-TOHIP is increased as 0.2370 . When the attacker nodes are present, the minimum delay of the proposed routing protocol is achieved as 0.2388 at the fixed transmission time of $2 \mathrm{~s}$.

\subsubsection{Performance Evaluation Based on Normalized Packet Drop}

Figure 6 shows the comparison results of the both TOHIP and FL-TOHIP using the losing rate of the transmission packets. When the data transmission time is set as $5 \mathrm{~s}$, the range of packet drop is obtained by TOHIP and FL-TOHIP as 0.203 and 0.200 , respectively. Here, the above results are obtained without considering any attacker nodes. From the results, we can say that the minimum number of packet drop is achieved by the proposed FL-TOHIP compared to the existing TOHIP. Then, the performance of the packet drop is analyzed with the MANET with attacker node. When the transmission time of data is fixed as $5 \mathrm{~s}$, the packet drop range of

A

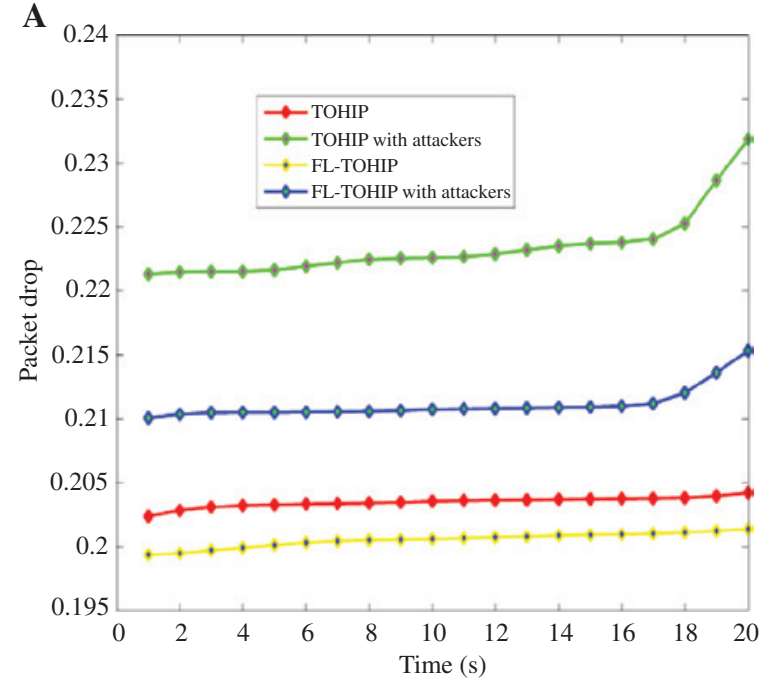

B

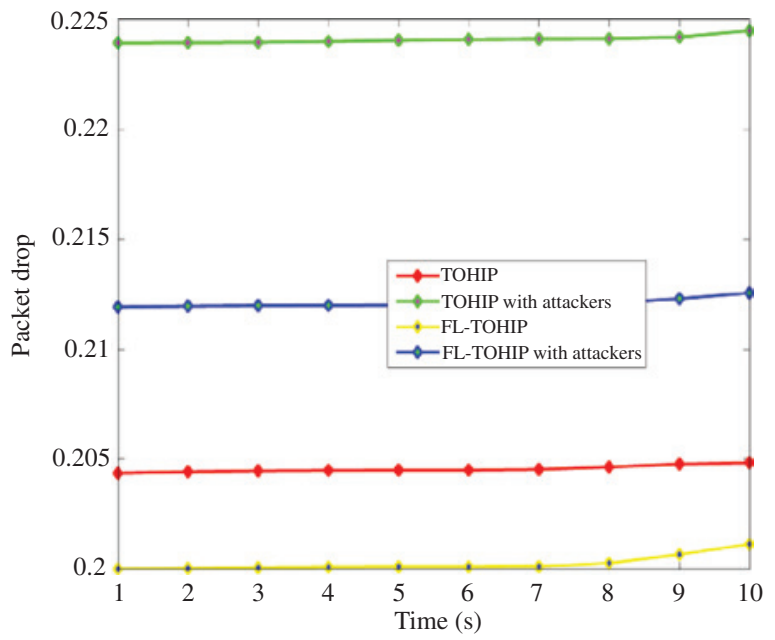

Figure 6: Packet Drop Analysis: (A) $t=20$ and Nodes $=100$ and (B) $t=10$ and Nodes $=100$. 
both TOHIP and FL-TOHIP is obtained as 0.221 and 0.210 , respectively. However, the proposed routing protocol achieved the minimum range of packet drop when compared to the existing protocol.

Figure 6B shows the rate of loss of packet analysis using the TOHIP and FL-TOHIP by considering the $100 \times 100 \mathrm{~m}$ MANET area. Initially, the normalized packet drop is measured using the routing protocol without the presence of the attacker node. At first, the transmission time of data packet is set as $2 \mathrm{~s}$. Then, the rate of packet drop of the proposed FL-TOHIP and TOHIP is obtained as 0.204 and 0.200 , respectively. By increasing the time of data transmission from 2 to $20 \mathrm{~s}$, the packet drop range of both proposed and TOHIP is achieved as 0.2048 and 0.201 , respectively. When the attacker node is available with the data transmission, the packet drop rate is again analyzed by both the TOHIP and FL-TOHIP. When the time of data transmission is set as $20 \mathrm{~s}$, the minimum rate of packet drop is achieved by the proposed FL-TOHIP compared to the existing TOHIP. At time $20 \mathrm{~s}$, the maximum packet drop is obtained by the TOHIP as 0.224 .

\section{Conclusion}

We have presented the FL-TOHIP to determine the optimal selected path. The main objective of this research work was to provide better transmission in MANETs using the optimal routing path. First, we used TOHIP to provide a different number of disjoint paths. Then, the TOHIP was performed through three phases, such as route request phase, route reply phase, and route probe phase. Thus, the multiple disjoint paths were obtained, which were then fed into the optimization algorithm. Second, the fractional lion algorithm was developed by combining the fractional theory and lion optimization algorithm. From the obtained disjoint paths, the optimal routing path was found by the fractional lion algorithm in which the lion updating was performed by fractional derivative. Thus, the proposed FL-TOHIP achieved the appropriate routing path for data transmission. The simulation results were validated and the performance was analyzed using throughput, delay, and normalized packet drop. The proposed algorithm attained the throughput value of 0.6, which ensured better data transmission capability.

\section{Bibliography}

[1] L. Abusalah, A. Khokhar and M. Guizani, A survey of secure mobile ad hoc routing protocols, IEEE Commun. Surv. Tutor. 10 (2008), 78-93.

[2] D. P. Agarwal and Q.-A. Zeng, Introduction to Wireless and Mobile Systems, 436 pp., Brooks/Cole Publishing, Andover, Hampshire, UK, ISBN No. 0534-408516, 2003.

[3] R. Biradar and S. Manvi, Review of multicast routing mechanisms in mobile ad hoc networks, Netw. Comput. Appl. 35 (2012), 221-239.

[4] R. C. Biradar and S. S. Manvi, Neighbor supported reliable multipath multicast routing in MANETs, Netw. Comput. Appl. 35 (2012), 1074-1085.

[5] A. Boukerche, B. Turgut, N. Aydin, Z. A. Mohammad, B. Ladislau and T. Damla, Routing protocols in ad-hoc networks a survey, Comput. Netw. 55 (2011), 3032-3080.

[6] V. R. Budyal and S. S. Manvi, ANFIS and agent-based bandwidth and delay aware anycast routing in mobile ad-hoc networks, Netw. Comput. Appl. 39 (2014), 140-151.

[7] T. Camp, J. Boleng and V. Davies, A survey of mobility models for ad hoc networks research, Wireless Commun. Mobile Comput. 2 (2002), 483-502.

[8] N. K. Cauvery and K. V. Viswanatha, Enhanced ant colony based algorithm for routing in mobile ad hoc network, Eng. Technol. World Acad. Sci. 46 (2008), 30-35.

[9] S. Chatterjee and S. Das, Ant colony optimization based enhanced dynamic source routing algorithm for mobile ad-hoc network, Inf. Sci. 295 (2015), 67-90.

[10] R. Kumar and D. Kumar, Multi-objective fractional artificial bee colony algorithm to energy aware routing protocol in wireless sensor network, Wireless Netw. 22 (2015), 1-14.

[11] R. Kumar and D. Kumar, Multi-objective fractional artificial bee colony algorithm to energy aware routing protocol in wireless sensor network, Wireless Netw. 22 (2016), 1461-1474. 
[12] P. Misra, Routing Protocols for Ad Hoc Mobile Wireless Networks, http://www.cse.wustl.edu/ jain/cis788-99/ftp/adhoc routing.

[13] S. Mueller, R. P. Tsang and D. Ghosal, Multipath routing in mobile ad hoc networks: issues and challenges, in: M. C. Calzarossa and E. Gelenbe, eds., Performance Tools and Applications to Networked Systems, Lecture Notes in Computer Science, vol. 2965, Springer, Berlin, Heidelberg, 2004.

[14] A. Munaretto and M. Fonseca, Routing and quality of service support for mobile ad hoc networks, Comput. Netw. 51 (2007), 3142-3156.

[15] B. Nancharaiah and B. Chandra Mohan, The performance of a hybrid routing intelligent algorithm in a mobile ad hoc network, Comput. Elect. Eng. 40 (2014), 1255-1264.

[16] B. R. Rajakumar, The lion's algorithm: a new nature-inspired search algorithm, Procedia Technol. 6 (2012), 126-135.

[17] F. Rookhosh et al., Disjoint categories in low delay and on-demand multipath dynamic source routing ad hoc networks, in: Proceedings of International Conference on Distributed Framework and Applications, pp. 207-213, 2008. https://web.njit. edu/ jt66/publications/paper_adhoc2014.pdf

[18] E. M. Royer and C.-K. Toh, A review of current routing protocols for ad hoc mobile wireless networks, IEEE Pers. Commun. 6 (1999), 46-55.

[19] D. Sharma, M. Yadav and H. Kumar, An on-demand energy efficient routing algorithm for mobile ad hoc networks, in: Proceedings of International Conference on Computing, Communication and Automation, vol. 2713, pp. 332-335, 2015.

[20] G. Singh, N. Kumar and A. K. Verma, Ant colony algorithms in MANETs: a review, Netw. Comput. Appl. 35 (2012), $1964-1972$.

[21] G. Singh, N. Kumar and A. K. Verma, OANTALG: an orientation based ant colony algorithm for mobile ad hoc networks, Wireless Personal Commun. 77 (2014), 1859-1884.

[22] S. Taheri, S. Hartung and D. Hogrefe, Anonymous group-based routing in MANETs, Inf. Security Appl. Arch. 22 (2015), 87-98.

[23] K. Tan, Q. Zhang and W. Zhu, Shortest path routing in partially connected ad hoc networks, in: Proceedings of International Conference on Global Telecommunications, vol. 2, pp. 1038-1042, 2003.

[24] H. Xu, X. Wu and S. Garcia-Luna, A unified analysis of routing in MANETs, IEEE Trans. Commun. 58 (2010), 911-922.

[25] Y. Zhang, G. Wang, Q. Hu, Z. Li and J. Tian, Design and performance study of a topology-hiding multi-path routing protocol for mobile ad hoc networks, in: Proceedings of IEEE Conference on Computer Communications, pp. 10-18, 2012.

[26] Y. Zhang, T. Yan, J. Tian, Q. Hu, G. Wang and Z. Li, TOHIP: a topology-hiding multipath routing protocol in mobile ad hoc networks, Ad Hoc Networks 21 (2014), 109-122. 